\title{
RIGOROUS GEOREFERENCING OF ALSAT-2A PANCHROMATIC AND MULTISPECTRAL IMAGERY
}

\author{
I. Boukerch ${ }^{\text {a }}$, M. Hadeid ${ }^{\text {b }}$, R. Mahmoudi ${ }^{\text {a }}$, B. Takarli ${ }^{\text {a }}$, \\ K. Hasni ${ }^{\mathrm{a}}$ \\ ${ }^{a}$ CTS, 01 avenue de Palestine, Arzew, Oran, Algeria - issam.boukerch@yahoo.fr \\ ${ }^{\mathrm{b}}$ Dept. of Geography, Oran University, Oran, Algeria.
}

ISPRS WG I/4, III/4, IC IV/VIII, VII/2

KEY WORDS: ALSAT-2A, multispectral, Panchromatic, Pushbroom sensor, Dimap, rigorous model, accuracy

\begin{abstract}
:
The exploitation of the full geometric capabilities of the High-Resolution Satellite Imagery (HRSI), require the development of an appropriate sensor orientation model. Several authors studied this problem; generally we have two categories of geometric models: physical and empirical models.

Based on the analysis of the metadata provided with ALSAT-2A, a rigorous pushbroom camera model can be developed. This model has been successfully applied to many very high resolution imagery systems. The relation between the image and ground coordinates by the time dependant collinearity involving many coordinates systems has been tested. The interior orientation parameters must be integrated in the model, the interior parameters can be estimated from the viewing angles corresponding to the pointing directions of any detector, these values are derived from cubic polynomials provided in the metadata. The developed model integrates all the necessary elements with 33 unknown. All the approximate values of the 33 unknowns parameters may be derived from the informations contained in the metadata files provided with the imagery technical specifications or they are simply fixed to zero, so the condition equation is linearized and solved using SVD in a least square sense in order to correct the initial values using a suitable number of well-distributed GCPs.

Using Alsat-2A images over the town of Toulouse in the south west of France, three experiments are done. The first is about 2D accuracy analysis using several sets of parameters. The second is about GCPs number and distribution. The third experiment is about georeferencing multispectral image by applying the model calculated from panchromatic image.
\end{abstract}

\section{INTRODUCTION}

Alsat-2A satellite was launched into orbit on 12 July 2010, from the launch site Sriharikota (India) by Indian PSLV-C15 launcher. It has five push broom sensors, panchromatic and multispectral in four bands namely blue, green, red and infrared. The panchromatic image is acquired with a spatial resolution of $2.5 \mathrm{~m}$ and a swath of $17.5 \mathrm{~km}$ at nadir. Multispectral bands have a resolution of $10 \mathrm{~m}$ with the same swath. ALSAT-2A takes images at an altitude of $680 \mathrm{~km}$ on a sun-synchronous orbit with an orbital period of 98.2 minutes and an orbital cycle of 29 days (ASAL, 2011; Kameche et al., 2011).

As part of the implementation of space applications projects in the National Space Program 2020 with the collaboration of all national users; the use of an Algerian very high resolution space system has a significant technological and economical interest, by reducing the use of international space systems products, thus reducing the projects costs(Boukerch et al., 2012)

Before using HRSI for GIS or mapping applications, we must, in first consider the geometric aspect of this satellite imagery product, this is equivalent to give a response to the question "how the imaging system transform the location of the pixels on the earth into the image?" (Boukerch and Bounour, 2006).

Several authors studied this problem; generally we have two categories of geometric models: physical and empirical models. The empirical, implicit or non parametric models can be used when the parameters of the acquisition systems or a rigorous 3D physical model are not available. Since they do not reflect the source of distortions (Toutin et al., 2002) these models represent the acquisition system as a mathematical transformation (such as rational functions, 2D or 3D polynomials) between object and image spaces. (Boukerch and Bounour, 2006; Chen et al., 2006; Fraser, 2003; Grodecki et al., 2003; Poli, 2005; Toutin, 2004).

The physical called also rigorous or deterministic models reflects the physical reality of the viewing geometry (platform, sensor, Earth and sometimes map projection); generally in the optical imagery these models are based on the well-known collinearity condition (Chen et al., 2005; Jung et al., 2007; Ke and Wolniewicz, 2005; Liu et al., 2011; Poli, 2005; Weser et al., 2008). As (Michalis and Dowman, 2008) mentioned, the pushbroom model is a kinematic model; Due to the dynamic nature of pushbroom imaging geometry, each framlet has its own exterior orientation parameters (EOP) and under the assumption that the images are acquired with a pushbroom scanner using a constant time interval. A time dependent collinearity equation can be developed.

There are two approaches for the EOP modelling (Jeong and Bethel, 2008; Kim and Dowman, 2006; Liu et al., 2011; Michalis and Dowman, 2008; Poli, 2005; Weser et al., 2008). The approach used in this study is to use the satellite orbital parameters (or position and velocity) and attitude angles as model parameters; the compensation of systematic errors inherent in vendor-supplied orientation data is achieved through a least squares sensor orientation adjustment, which incorporates additional parameters for bias compensation and 
employs a modest number of ground control points (Liu et al., 2011).

The interior orientation parameters (IOP) are generally directly given in the sensor specifications as the principal point position, focal length and distortions. The IOPs can also be provided in indirect way (such for SPOT5), through the definition of two orthogonal viewing angles for each pixel of the CCD array, which define a bundle of rays between the projection centre and the pixel centres of the CCD array (Weser et al., 2008). In a similar way THEOS, ALSAT-2A and other platforms represent the IOPs through view angle values that are provided in the metadata for each detector in the CCD array in the form of cubic polynomials (Liu et al., 2011).

\section{ALSAT-2A AUXILIARY DATA}

The metadata file give us the orbit, attitude and the camera parameters data. These metadata are similar to SPOT5, Formosat2 and Theos metadata (Liu et al., 2010)

The orbit data are measured with a frequency of $1 \mathrm{~Hz}$ and are presented as a Time with a microsecond precision expressed in UTC time. The location vector is in meters in the Earth Centered Fixed (ECF) reference frame and the velocity vector in meters per second. The attitude data are measured with a frequency of $4 \mathrm{~Hz}$ and presented as attitude quaternion of the Attitude Orbit Control System reference frame tied in the spacecraft according to the ECI (J2000) reference frame. To compute J2000 referential to ITRF terrestrial referential rotation matrix, the $\mathrm{U}$ and $\mathrm{V}$ angels are also given in the metadata (ASAL, 2011).

The camera parameters are the instrument look angles in the camera frame provided in the form of cubic polynomials with the bias angles: roll, pitch and yaw. These angles define the camera frame orientation according to the Attitude Orbit Control System reference frame (Liu et al., 2010).

Other important information for the geometric model can be found in the metadata which are the reference line, reference time, line period and the number of rows. Also Yaw, Pitch and Roll angles that define the line of sight reference frame orientation according to the Platform coordinate system.

\section{RIGOROUS SENSOR ORIENTATION MODEL}

A polar orbital satellite usually moves along a well-defined close-to-circular elliptical orbit. All scan line exposure stations would therefore be constrained on this orbit path. For a short arc, the assumption of a "two-body" orbit may be used. This may be parameterized with six elements of a state vector or, equivalently, six Kepler elements (Jeong and Bethel, 2008; Jeong, 2008; Jung et al., 2007)

The initial sensor attitude can be assumed as a nadir looking but due to the agility, an interpolation between the start and the end attitudes is more appropriate, so, for each scan line, the interpolated attitude from given start and end attitude will be assigned (Jeong and Bethel, 2008; Jeong, 2008).

Based on the analysis of the metadata provided with ALSAT$2 \mathrm{~A}$, a rigorous pushbroom camera model can be developed.
This model has been successfully applied to many very high resolution imagery systems (Chen et al., 2006, 2005; Jung et al., 2007; Ke and Wolniewicz, 2005; Poli, 2005; Weser et al., 2008).

The relation between points in an earth-centered coordinate system to its projected point in the image coordinate system can be expressed as (Boukerch et al., 2012; Jeong, 2008):

$P_{\text {img }}=\lambda M_{r}\left[P_{\text {grd }}-\left[M_{\text {orb-itrf }}^{T}\left(P_{K}+P_{c}\right)\right]\right]$

Where $\mathrm{P}_{\text {img }}$ is the image coordinates vector, $\mathrm{P}_{\text {grd }}$ is the ground coordinates vector, $\mathrm{P}_{\mathrm{K}}$ is the vector from earth centre to satellite in the orbit plane, $\mathrm{M}_{\text {orb-itrf }}$ is the rotation matrix applied to ECEF coordinates to bring them parallel to the orbital coordinates system, $\mathrm{P}_{\mathrm{c}}$ is the position correction vector, $\mathrm{M}_{\mathrm{r}}$ is a rotation matrix from ECEF to the camera frame and $\lambda$ is a scale factor (see Figure 1).

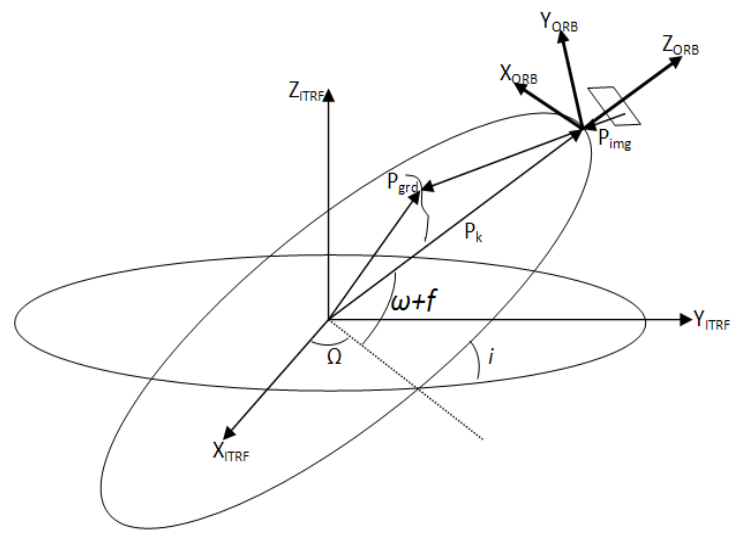

Figure 1. Geometrical relationship between image and ground points.

\section{TEST DATASET}

The dataset is composed of panchromatic images of $1 \mathrm{~A}$ processing level over Toulouse town in south west of France. The first image is taken on 07 February 2011 with viewing angles of -8.785521 and 0.396593 degrees cross and along track respectively. This image is equipped with 27 points; the second image is taken on 04 February 2011 with viewing angles of 15.432805 and 5.577266 degrees cross and along track. The terrain is relatively flat with elevations are between 130 to 250 $\mathrm{m}$. This image is equipped with 22 points collected from cadastral plans of Toulouse town using Géopotail 3D mapping service. 
International Archives of the Photogrammetry, Remote Sensing and Spatial Information Sciences, Volume XL-1/W1, ISPRS Hannover Workshop 2013, 21 - 24 May 2013, Hannover, Germany

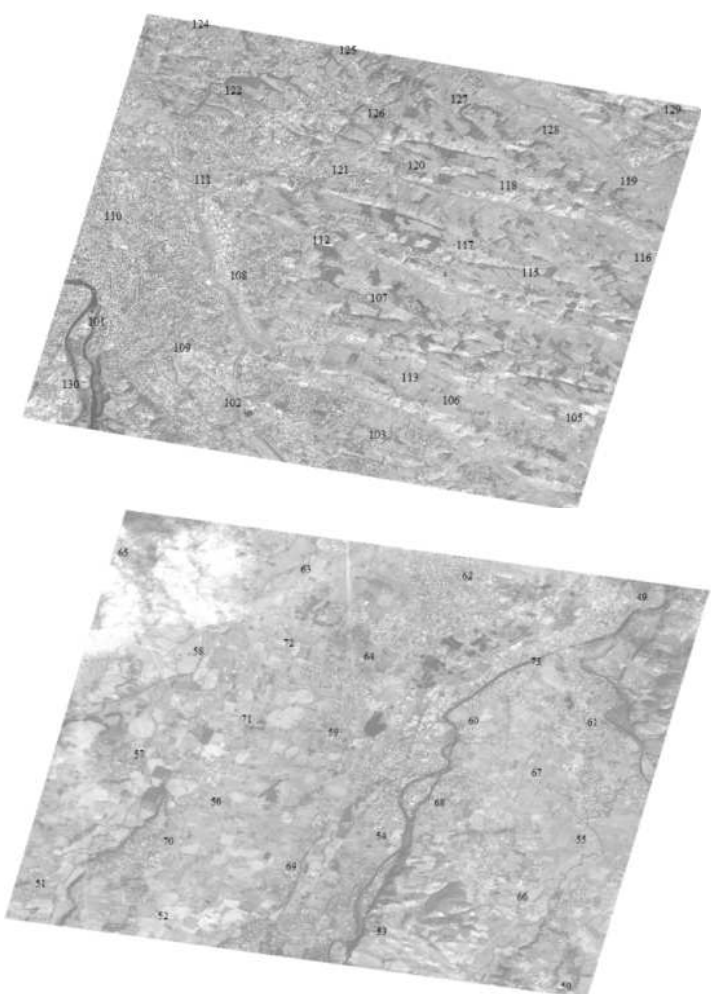

Figure 2. Toulouse images and GCPs distribution.

\section{EXPERIMENTAL RESULTS}

In this study only $2 \mathrm{D}$ accuracy analysis is possible, due to the single image coverage. The check point accuracy is estimated in $2 \mathrm{D}$ via a back projection of $3 \mathrm{D}$ check point coordinates into image space using the adjusted orientation parameters. It is also possible to check the accuracy by the use of a forward projection to an established height value in object space(Liu et al., 2011).

\subsection{D accuracy analysis}

The first georeferencing experiment is about 2D accuracy analysis using the Toulouse dataset. in this test eleven well distributed GCP's on each image are used to estimate several sets of parameters, the remaining points are considered as check points.

The developed model based on eq. 1 uses second order polynomial function of time for the modelling of the errors in the position and the attitude of the satellite. The developed program offers the possibility to changing the orders of the polynomials, so different sets of variables are tested in this experiment. In the tables 3 and 4 we denote by X Y Z the translations parameters, $\Omega \Phi \mathrm{K}$ the rotations about $\mathrm{X} \mathrm{Y} \mathrm{Z} \mathrm{axis}$ successively and the number represent the order of the polynomial.

\begin{tabular}{|c|c|c|c|c|}
\hline \multirow{2}{*}{$\begin{array}{c}\text { Used } \\
\text { parameters }\end{array}$} & \multicolumn{2}{|c|}{$\begin{array}{c}\text { RMS of residuals } \\
\text { on GCP (pixel) }\end{array}$} & \multicolumn{2}{c|}{$\begin{array}{c}\text { RMS of residuals } \\
\text { on CP (pixel) }\end{array}$} \\
\cline { 2 - 5 } & $\mathrm{X}$ & $\mathrm{Y}$ & $\mathrm{X}$ & $\mathrm{Y}$ \\
\hline $\mathrm{X} \mathrm{Y} \mathrm{Z} \Omega \Phi$ & 10.456 & 14.162 & 10.308 & 10.199 \\
$\mathrm{~K}$ & & & & \\
\hline $\mathrm{X} 2 \mathrm{Y} 2 \mathrm{Z} 2$ & 0.335 & 0.669 & 0.646 & 0.766 \\
$\Omega 2 \Phi 2 \mathrm{~K} 2$ & & & & \\
\hline
\end{tabular}

\begin{tabular}{|c|c|c|c|c|}
\hline $\mathrm{X} 2 \mathrm{Y} 2 \mathrm{Z} 2$ & 2.699 & 0.795 & 1.574 & 0.819 \\
\hline$\Omega 2 \Phi 2 \mathrm{~K} 2$ & 0.326 & 3.687 & 0.702 & 2.598 \\
\hline $\begin{array}{c}\mathrm{X} 2 \mathrm{Y} 2 \mathrm{Z} 2 \\
\Omega\end{array}$ & $1.425 \mathrm{~K}$ & 0.739 & 0.919 & 0.860 \\
\hline $\begin{array}{c}\mathrm{X} \mathrm{Y} \mathrm{Z} \Omega 2 \\
\Phi 2 \mathrm{~K} 2\end{array}$ & 0.306 & 1.194 & 0.676 & 0.893 \\
\hline $\begin{array}{c}\mathrm{X} 2 \mathrm{Y} 2 \mathrm{Z} 2 \\
\mathrm{~K} 2\end{array}$ & 0.328 & 0.799 & 0.701 & 0.813 \\
\hline $\begin{array}{c}\mathrm{Z} 2 \Omega 2 \Phi 2 \\
\mathrm{~K} 2\end{array}$ & 0.329 & 0.805 & 0.702 & 0.809 \\
\hline $\begin{array}{c}\mathrm{Z} 1 \Omega 2 \Phi 2 \\
\mathrm{~K} 1\end{array}$ & 0.340 & 0.803 & 0.689 & 0.812 \\
\hline $\begin{array}{c}\mathrm{Z} 1 \Omega 2 \Phi 2 \\
\mathrm{~K}\end{array}$ & 1.612 & 0.802 & 0.959 & 0.808 \\
\hline $\begin{array}{c}\mathrm{Z} \Omega 2 \Phi 2 \\
\mathrm{~K}\end{array}$ & 1.611 & 1.240 & 0.959 & 0.906 \\
\hline $\begin{array}{c}\mathrm{Z} 1 \Omega 2 \Phi 2 \\
\mathrm{~K} 2\end{array}$ & 0.329 & 0.803 & 0.702 & 0.810 \\
\hline $\begin{array}{c}\mathrm{Z} \Omega 2 \Phi 2 \\
\mathrm{~K} 2\end{array}$ & 0.330 & 1.240 & 0.704 & 0.907 \\
\hline $\begin{array}{c}\mathrm{Z} \Omega 2 \Phi 2 \\
\mathrm{~K} 1\end{array}$ & 0.341 & 1.241 & 0.688 & 0.909 \\
\hline $\begin{array}{c}\mathrm{Z} \Omega 1 \Phi 2 \\
\mathrm{~K} 1\end{array}$ & 0.4540 & 9.4277 & 0.7737 & 7.1067 \\
\hline $\begin{array}{c}\mathrm{Z} \Omega 2 \Phi 1 \\
\mathrm{~K} 1\end{array}$ & 1.5293 & 0.8036 & 1.4103 & 0.8126 \\
\hline
\end{tabular}

Table 3. Results for different sets of parameters using the first Toulouse image.

\begin{tabular}{|c|c|c|c|c|}
\hline \multirow{2}{*}{$\begin{array}{c}\text { Used } \\
\text { parameters }\end{array}$} & \multicolumn{2}{|c|}{$\begin{array}{c}\text { RMS of residuals } \\
\text { on GCP (pixel) }\end{array}$} & \multicolumn{2}{c|}{$\begin{array}{c}\text { RMS of residuals } \\
\text { on CP (pixel) }\end{array}$} \\
\cline { 2 - 5 } $\mathrm{X}$ & $\mathrm{Y}$ & $\mathrm{X}$ & $\mathrm{Y}$ \\
\hline $\begin{array}{c}\mathrm{X} \mathrm{Y} \mathrm{Z} \Omega \Phi \\
\mathrm{K}\end{array}$ & 7.982 & 5.465 & 3.321 & 3.460 \\
\hline $\begin{array}{c}\mathrm{X} 2 \mathrm{Y} 2 \mathrm{Z} 2 \\
\Omega 2 \Phi 2 \mathrm{~K} 2\end{array}$ & 0.380 & 0.350 & 0.886 & 0.808 \\
\hline $\mathrm{X} 2 \mathrm{Y} 2 \mathrm{Z} 2$ & 2.883 & 0.739 & 2.160 & 0.935 \\
\hline$\Omega 2 \Phi 2 \mathrm{~K} 2$ & 0.426 & 6.823 & 0.921 & 4.553 \\
\hline $\begin{array}{c}\mathrm{X} 2 \mathrm{Y} 2 \mathrm{Z} 2 \\
\Omega \Phi \mathrm{K}\end{array}$ & 1.699 & 0.736 & 1.027 & 0.955 \\
\hline $\begin{array}{c}\mathrm{X} \mathrm{Y} \mathrm{Z} \Omega \\
\Phi 2 \mathrm{~K} 2\end{array}$ & 0.398 & 0.798 & 0.902 & 0.946 \\
\hline $\begin{array}{c}\mathrm{X} 2 \mathrm{Y} 2 \mathrm{Z} 2 \\
\mathrm{~K} 2\end{array}$ & 0.429 & 0.739 & 0.920 & 0.932 \\
\hline $\begin{array}{c}\mathrm{Z} 2 \Omega 2 \Phi 2 \\
\mathrm{~K} 2\end{array}$ & 0.430 & 0.743 & 0.920 & 0.921 \\
\hline $\begin{array}{c}\mathrm{Z} 1 \Omega 2 \Phi 2 \\
\mathrm{~K} 1\end{array}$ & 0.430 & 0.741 & 0.920 & 0.924 \\
\hline $\begin{array}{c}\mathrm{Z} 1 \Omega 2 \Phi 2 \\
\mathrm{~K}\end{array}$ & 1.906 & 0.745 & 1.066 & 0.922 \\
\hline $\begin{array}{c}\mathrm{Z} \Omega 2 \Phi 2 \\
\mathrm{~K}\end{array}$ & 1.899 & 0.806 & 1.065 & 0.926 \\
\hline $\begin{array}{c}\mathrm{Z} 1 \Omega 2 \Phi 2 \\
\mathrm{~K} 2\end{array}$ & 0.430 & 0.741 & 0.920 & 0.924 \\
\hline $\begin{array}{c}\mathrm{Z} \Omega 2 \Phi 2 \\
\mathrm{~K} 2\end{array}$ & 0.430 & 0.806 & 0.920 & 0.927 \\
\hline $\begin{array}{c}\mathrm{Z} 22 \Phi 2 \\
\mathrm{~K} 1\end{array}$ & 0.430 & 0.806 & 0.920 & 0.927 \\
\hline $\begin{array}{c}\mathrm{Z} \Omega 1 \\
\mathrm{~K} 1\end{array}$ & 0.431 & 1.008 & 0.920 & 1.009 \\
\hline $\begin{array}{c}\mathrm{Z} \Omega 2 \Phi 1 \\
\mathrm{~K} 1\end{array}$ & 3.519 & 0.748 & 1.727 & 0.923 \\
\hline
\end{tabular}

Table 4. Results for different sets of parameters using the second Toulouse image. 
The first set of parameters ( $\mathrm{X} \mathrm{Y} \mathrm{Z} \Omega \Phi \mathrm{K}$ ) allows the representation of translation and attitude as constant correction to the parameters derived from metadata. The resulting image point discrepancies lay between 3 and 15 pixels. The full parameters set (X2 Y2 Z2 $\Omega 2 \Phi 2 \mathrm{~K} 2$ ) provide the best results for this dataset where the RMSE on check points is less than 1 pixel. It is noteworthy that the sets based on higher order attitudes provide better results than those based on translations.

Due to the existing correlation between parameters, we used two groups of parameters sets X Y Z K and Z $\Omega \Phi \mathrm{K}$ with different orders of each parameter(Jeong and Bethel, 2008; Jeong, 2008). We note that the use of second order polynomial for attitudes with second order polynomial for $\mathrm{Z}$ translation (Z2 $\Omega 2 \Phi 2 \mathrm{~K} 2$ ) provides similar results as the full parameters set. The application of a constant correction to $\mathrm{Z}$ translation and linear correction of the $\mathrm{K}$ attitude parameter ( $\mathrm{Z} \Omega 2 \quad \Phi 2 \mathrm{~K} 1$ ) ensure a sub pixel positional accuracy.

\subsection{Points number and distribution}

In this section we test the Influence of the ground control point number and their distribution on the accuracy of the calculated model. For this test, only the first image of Toulouse is used.

The model is calculated using different combinations of GCPs. the first set results from the various combinations of 2 GCPs that can be taken among the 27 available points and the 25 remaining points are used as check points; this leads to 351 possible combinations. The other sets results from changing the GCPs number from 2 to 14 ; due to the higher number of possible combinations when using 3 to 14 GCPs we choose 2000 combinations randomly among all possible ones.

For each used combination of GCPs in the set, the RMSE of the resulting model is calculated using check points. We calculate also the standard deviation and the mean of the $\mathrm{X}$ and $\mathrm{Y}$ coordinates of GCPs. Then for the whole set we calculate the correlation between the RMSEs and the standard deviation on the one hand and the correlation between the RMSEs and the mean on the other hand. The standard deviation and the mean of the GCPs coordinates give an indication about the GCPs distribution. Also we calculate the minimum RMSE, percentiles of the RMSEs of the sets at $50 \%$ and $95 \%$ and the percentage of RMSEs of less than 1 pixel for each set. The results are presented in Table 5 .
When using 2 points as GCPs, the best achievable accuracy is 8.47 pixels. Using 4 or more GCPs, sub pixel accuracy can be achieved. this can be explained by the fact that there are seven or eight uncorrelated parameters among all used parameters. The points must have a maximum spacing in $\mathrm{Y}$ direction because of the relatively higher correlation between RMSE and standard deviation of the Y coordinate of the GCPs, this can be explained by the image capture mode which is a pushbroom moving along the $\mathrm{Y}$ axis. the position of the barycentre of the points is not very important since the correlation between the mean of the $\mathrm{X}$ and $\mathrm{Y}$ coordinates of the GCPs and the RMSE is very low. The minimum RMSE resulting from the best distribution become stable around 0.3 pixel using more than 10 GCPs.

\subsection{Georeferencing multispectral image}

This third experiment is about georeferencing multispectral image. We use the result provided by the panchromatic image model which is calculated using eleven points as control points and the second order polynomial correction for all the position and attitude parameters.

The two images (panchromatic and multispectral) are taken with a lapse of time of 0.060788 second due to the shift of the two detectors lines in the focal plan. The major differences between the panchromatic and multispectral images in the metadata are the LOS coefficients, the reference time which is the time of capture of the first image line and the line period.

All the points on the multispectral image are considered as check points; the sample coordinate of each point on the image is used to calculate the image coordinates by the mean of LOS coefficients. The line coordinate is utilized with line period and reference time to calculate the time of acquisition of the point, using this time we can obtain the corrections to the initial position and attitude based on the model calculated from the panchromatic image.

The terrain coordinates of the points are integrated to the time dependant collinearity (see Eq 1) and the image coordinates are calculated through a back projection. The comparison between the observed image coordinates and those obtained by back projection permit to estimate the accuracy where the standard deviation is 0.6251 and 0.8068 pixel in $\mathrm{x}$ and y respectively for the entire set of points on the multispectral image.

\begin{tabular}{|c|c|c|c|c|c|c|c|c|}
\hline $\begin{array}{c}\text { GCPs } \\
\#\end{array}$ & Corr $\sigma \mathrm{x}$ & Corr $\sigma \mathrm{y}$ & Corr $\mu \mathrm{x}$ & Corr $\mu \mathrm{y}$ & min RMSE & RMSE at 50\% & RMSE at 95\% & $\begin{array}{c}\% \text { of RMSE < } \\
\text { pixel }\end{array}$ \\
\hline 2 & 0.34 & -0.74 & 0.08 & -0.18 & 8.47 & 36.58 & 98.34 & 0.00 \\
\hline 3 & -0.03 & -0.58 & 0.05 & -0.17 & 5.29 & 18.09 & 59.93 & 0.00 \\
\hline 4 & 0.13 & -0.42 & 0.07 & -0.26 & 0.52 & 5.19 & 21.48 & 3.00 \\
\hline 5 & -0.07 & -0.33 & 0.08 & -0.14 & 0.41 & 2.65 & 26.86 & 12.60 \\
\hline 6 & -0.05 & -0.35 & 0.07 & -0.15 & 0.60 & 2.99 & 18.80 & 6.45 \\
\hline 7 & -0.08 & -0.41 & 0.14 & -0.15 & 0.48 & 2.60 & 17.27 & 8.35 \\
\hline 8 & -0.11 & -0.42 & 0.05 & -0.12 & 0.39 & 1.79 & 10.71 & 18.05 \\
\hline 9 & -0.07 & -0.39 & 0.00 & -0.17 & 0.37 & 1.49 & 8.38 & 26.45 \\
\hline 10 & -0.09 & -0.44 & 0.01 & -0.12 & 0.37 & 1.16 & 5.58 & 40.05 \\
\hline 11 & -0.08 & -0.41 & -0.05 & -0.07 & 0.30 & 1.05 & 4.55 & 46.95 \\
\hline 12 & -0.08 & -0.37 & -0.06 & -0.05 & 0.30 & 0.91 & 3.68 & 56.90 \\
\hline 13 & -0.13 & -0.32 & -0.06 & 0.02 & 0.31 & 0.85 & 2.79 & 64.70 \\
\hline 14 & -0.10 & -0.29 & -0.07 & 0.04 & 0.30 & 0.79 & 2.76 & 69.50 \\
\hline
\end{tabular}

Table 5. The correlation between GCPs distribution and the resulting accuracy using different GCPs number 


\section{CONCLUSION}

The parameters integrated in the metadata provided with the ALSAT2-A permit the development of a rigorous sensor model.

The developed program offers the possibility to change the orders of the correction polynomials and also the variables selection, so different sets of variables are tested; the use of the second order polynomials for attitude and position correction gives 0.646 and 0.766 as RMS of residuals on check points.

The points number and distribution is studied through the correlation between the RMSE and the standard deviation on one hand and RMSE with the mean of the $\mathrm{X}$ and $\mathrm{Y}$ coordinates of GCPs on the other hand. Using 4 or more GCPs sub pixel accuracy can be achieved. The points must have a maximum spacing in $\mathrm{Y}$ direction, the position of the barycentre of the points is not very important since the correlation between the mean of the $\mathrm{X}$ and $\mathrm{Y}$ coordinates of the GCPs and the RMSE is very low. The minimum RMSE resulting from the best distribution become stable around 0.3 pixel using more than 10 GCPs.

Considering the differences between multispectral and the panchromatic, the model calculated using the panchromatic image is used to estimate the accuracy of the multispectral image; the standard deviation is 0.6251 and 0.8068 pixel in $\mathrm{x}$ and $y$ of entire set of points on the multispectral image.

\section{ACKNOWLEDGEMENTS}

The authors are grateful to M. F. Benhammouda and A. Botte from Algerian Space Agency for their help.

\section{REFERENCES}

ASAL, 2011. 2A, Alsat-2A images technical specification.

Boukerch, I., Bounour, H., 2006. Geometric modelling and orthorectification of SPOTs super mode images. Revue française de photogrammétrie et de télédétection 61-65.

Boukerch, I., Hadied, M., Mahmoudi, R., Takarli, B., Hasni, K., 2012. Rigorous geometrical modeling of ALSAT-2A Algerian satellite, in: SPIE Remote Sensing. International Society for Optics and Photonics, p. 85331V-85331V-9.

Chen, L., Teo, T., Liu, C., 2006. The geometrical comparisons of RSM and RFM for FORMOSAT-2 satellite images. Photogrammetric engineering and remote sensing 72, 573.

Chen, L., Teo, T., Liu, L., 2005. Rigorous georeferencing for Formosat-2 satellite images by least squares collocation, in: International Geoscience and Remote Sensing Symposium. p. 3526 .

Fraser, C.S., 2003. Prospects for mapping from high-resolution satellite imagery. Asian Journal of Geoinformatics 4, 3-10.

Grodecki, J., Dial, G., Lutes, J., 2003. Error propagation in block adjustment of high-resolution satellite images, in: ASPRS 2003 Annual Conference Proceedings. pp. 5-9.
Jeong, I., Bethel, J., 2008. TRAJECTORY MODELING FOR SATELLITE IMAGE TRIANGULATION. the International Archives of the Photogrammetry, Remote Sensing and Spatial Information Sciences, Beijing.

Jeong, I.-S., 2008. Resolving parameter dependencies in satellite sensor models. Phd Diss, Purdue University, Purdue.

Jung, H., Kim, S., Won, J., Lee, D., 2007. Line-of-Sight Vector Adjustment Model for Geopositioning of SPOT-5 Stereo Images. Photogrammetric Engineering and Remote Sensing 73, 1267.

Kameche, M., Gicquel, A.H., Joalland, D., 2011. ALSAT-2A TRANSFER AND FIRST YEAR OPERATIONS. Journal of Aerospace Engineering 3, 67.

Ke, L.C., Wolniewicz, W., 2005. Very High Resolution Satellite Image Triangulation. Presented at the Asian Conference on Remote Sensing, Hanoi, Vietnam.

Kim, T., Dowman, I., 2006. Comparison of two physical sensor models for satellite images: position-rotation model and orbitattitude model. The Photogrammetric Record 21, 110-123.

Liu, S., Fraser, C.S., Zhang, C., Ravanbakhsh, M., Tong, X., 2010. Geometric Accuracy Evaluation Of Theos Imagery. Presented at the Asian Conference on Remote Sensing, Hanoi, Vietna.

Liu, S., Fraser, C.S., Zhang, C., Ravanbakhsh, M., Tong, X., 2011. Georeferencing performance of THEOS satellite imagery. The Photogrammetric Record 26, 250-262.

Michalis, P., Dowman, I., 2008. A generic model for along track stereo sensors using rigorous orbit mechanics. Photogrammetric Engineering and Remote Sensing 74, 303.

Poli, D., 2005. Modelling of spaceborne linear array sensors. Diss., Technische Wissenschaften, Eidgenössische Technische Hochschule ETH Zürich, Nr. 15894, 2005.-Ref.: Armin Grün; Korref.: Ian Dowman.

Toutin, T., 2004. Review article: Geometric processing of remote sensing images: models, algorithms and methods. International Journal of Remote Sensing 25, 1893-1924.

Toutin, T., Chénier, R., Carbonneau, Y., 2002. 3D models for high resolution images: examples with QuickBird, IKONOS and EROS. INTERNATIONAL ARCHIVES OF PHOTOGRAMMETRY REMOTE SENSING AND SPATIAL INFORMATION SCIENCES 34, 547-551.

Weser, T., Rottensteiner, F., Willneff, J., Poon, J., Fraser, C.S., 2008. Development and testing of a generic sensor model for pushbroom satellite imagery. The Photogrammetric Record 23, 255-274. 